\section{PSQ-020 ANALYSIS OF THE EFFECTIVENESS OF SECUKINUMAB AND IXEKIZUMAB IN THE TREATMENT OF MODERATE-SEVERE PSORIASIS}

MDV Sanchez Matamoros Piazza, A Varas Perez, C Puivecino Moreno*, C Cuadros Martinez. Hospital Universitario Jerez De La Frontera, Pharmacy Services, 11407, Spain

\subsection{6/ejhpharm-2020-eahpconf.337}

Background and importance There are currently two drugs with the same mechanism of action, inhibitors of interleukin 17 (anti-IL-17), for the treatment of moderate-severe psoriasis. Aim and objectives To evaluate the efficacy of secukinumab and ixekizumab in terms of psoriasis area severity index (PASI) and dermatology life quality index (DLQI) in the treatment of moderate-severe psoriasis

Material and methods A retrospective observational study was conducted in patients treated with secukinumab and ixekizumab from February 2016 to October 2019. The variables collected were sex, diagnosis and previous biological treatment. The variation in PASI and DLQI were studied as the main efficacy variables. Data were obtained from the record of dispensation of outpatients and the electronic medical history.

Results Eighty-four patients were included, 44\% were men. In $50 \%$ of cases the anti-IL-17 drug was used as the firstline biological treatment, in $27 \%$ of cases as the secondline, in $6 \%$ as the thirdline and in $7 \%$ as the fourthline or successive treatment. The baseline average PASI was $6.87(\mathrm{SD}=3.5)$ and the average DLQI was $7.07(\mathrm{SD}=3.73)$. Twenty-one patients could not be evaluated due to lack of data recorded after the start of the anti-IL-17 drug. The percentage of patients with a reduced PASI was $9.52 \%, 19.05 \%$ and $44.44 \%$ for PASI $75 /$ 90/100, respectively: $63.16 \%$ obtained a DLQI after the start of treatment of $0-1$.

Conclusion and relevance Secukinumab and ixekizumab demonstrated effectiveness, representing a good therapeutic option for moderate to severe plaque psoriasis, including in both naive and patients refractory to other biological treatments. It is necessary to continue monitoring these patients to study the long term results.

\section{REFERENCES AND/OR ACKNOWLEDGEMENTS}

No conflict of interest.

\section{PSQ-021 TRANSVAGINAL MESH IN PELVIC ORGAN PROLAPSES: 2017-2019 RETROSPECTIVE ANALYSIS}

L Le Meur*, B Benoit, R Batista. Cochin Hospital, Pharmacy, Paris, France

\subsection{6/ejhpharm-2020-eahpconf.338}

Background and importance Over the past decade, there have been many discussions about vaginal mesh used for transvaginal repair of pelvic organ prolapse and the complications related to mesh procedures. It appears that mesh products probably entered the marked with too little information on their safety. On 16 April 2019, the FDA asked for immediate withdrawal of mesh used in the USA in these surgical procedures. Our country was affected by the same mesh withdrawals and the few mesh prostheses still marketed, reclassified as class III devices, are currently being re-evaluated. Even though very few mesh complications have been reported and despite the known side effects, some gynaecologists maintain there is need for such devices.
Aim and objectives Our study was a retrospective analysis of the records of patients treated for transvaginal prolapse of the pelvic organs by Ingynious prosthesis (AMI, Austria), mesh authorised and used in our hospital.

Material and methods The records of patients who underwent procedures between January 2017 and July 2019 were analysed. Justification for prosthesis placement, complications and post-surgery follow-up were analysed.

Results The average age of the 28 patients was 69.8 years and average BMI was $25 \mathrm{~kg} / \mathrm{m}^{2}$. Mesh placement decision was guided by patient risk factors (multiple surgeries, obesity, advanced age) in conjunction with risks linked to general anaesthesia. Ten patients $(35.7 \%)$ had suffered from pelvic prolapse recurrence, five after promotofixation and five after the use of pessaries. The only peroperative complications reported were two cases of bladder injury. Two cases of mesh over tension were described, and one required reoperation 2 days later. At that time, no serious complications were reported except a mesh cut detected a few days after placement, leading to a new procedure.

Conclusion and relevance This retrospective study confirmed vaginal meshes are used in well defined circumstances when promotofixation is contraindicated. This work needs to be continued to identify late complications. such as erosion. It is not known whether our regulatory authorities will continue to allow the use of these devices. However, when used wisely, according to each patient's history and by experienced surgeons, vaginal mesh placement is still an option to be considered.

\section{REFERENCES AND/OR ACKNOWLEDGEMENTS}

No conflict of interest.

\section{PSQ-022 ANALYSIS OF INFECTIONS ASSOCIATED WITH CENTRAL VENOUS CATHETERS USED FOR ADMINISTRATION OF PARENTERAL NUTRITION IN A THIRD LEVEL HOSPITAL}

MC Conde García*, MM Alañón Pardo, MT Gómez Lluch, A Pérez Facila, C Notario Dongil, JC Valenzuela Gámez. HG La Mancha Centro, Pharmacy, Alcazar De San Juan, Spain

\subsection{6/ejhpharm-2020-eahpconf.339}

Background and importance Central venous catheters (CVC) are devices used to draw blood and give treatments, including intravenous fluids and parenteral nutrition (PN). Among the side effects, bloodstream infections (BSIs) are considered to be the most severe complications, with a significant increase in morbidity and mortality.

Aim and objectives To determine the rate of catheter related bacteraemia (CRB) in hospitalised patients receiving central parenteral nutrition $(\mathrm{CPN})$ and to determine the relationship to type of canalised route.

Material and methods A prospective study was conducted in a third level hospital from 1 January 2016 to 30 June 2019. All admitted patients who received CPN were included. Data registered were hospitalisation unit, type of canalised route, days with $\mathrm{CVC}$ and isolated microorganisms in case of infections. The infection rate used was CRB/1000 days of CVC.

Results During the study period, 525 CVC were analysed in 428 patients: $76.6 \%$ were inserted in the operation room, $18.3 \%$ in the intensive care unit (ICU) and $5.1 \%$ in the hospitalisation room. The most common access was the jugular 
vein $(57.3 \%)$ followed by the subclavian vein $(34.5 \%)$, peripherally inserted central catheter (PICC, 7.6\%) and femoral vein (0.6\%). A total of 143 CVC (27.2\%) were removed for suspected BSIs, of which $50.3 \%$ were negative. There were 13 colonisations $(2.5 \%$ of the total), $38 \mathrm{CRB}(7.2 \%)$ and 20 positive results for central blood cultures without peripheral blood cultures $(3.8 \%)$, so it was not possible to determine whether it was colonisation or CRB. Regarding location, $54.9 \%$ of the infected CVC were jugular, 35.2\% subclavian and 9.9\% PICC. The overall CRB rate was 6.8. Results by services were: 4.7 in surgery services, 8.2 in the ICU and 11.0 in medical services. Staphylococcus was the most common isolate $(80.6 \%)$ followed by Escherichia coli and other gram negative bacteria (9.7\%). There were two infections caused by Acinetobacter (2.8\%) and three caused by Candida (4.2\%).

Conclusion and relevance Most of CVC had been inserted in the operation room and the most common access was the jugular vein. Half of the removed CVC for suspected BSIs were negative. The CRB rate in our centre could be underestimated because peripheral blood cultures were not extracted in a high number of cases. The microorganisms isolated in this study were similar to those found in the existing literature.

\section{REFERENCES AND/OR ACKNOWLEDGEMENTS}

1. Seisdedos Elcuaz R, Conde García MC, et al. Infecciones relacionadas con el CVC en pacientes con NPT. Nutr Hosp 2012 June (citado 2019 Sep 08);27:775-780.

No conflict of interest.

\section{PSQ-023 EFFECTIVENESS OF CEFTAZIDIME-AVIBACTAM IN INFECTIONS BY MULTIRESISTANT MICROORGANISMS}

CL Díaz Díaz, A Ferrer Machín*, E Tévar Alfonso, M Vera Cabrera, I González García, I Plasencia García, T Betancor García, J Merino Alonso. Hospital Universitario Nuestra Señora De La Candelaria, Hospital Pharmacy, Santa Cruz De Tenerife, Spain

\subsection{6/ejhpharm-2020-eahpconf.340}

Background and importance The acquisition of resistance by bacteria has meant that new antimicrobials appear. Ceftazidime-avibactam is a restricted antibiotic that is used in multiresistant infections that put the patient's life at risk.

Aim and objectives To evaluate the effectiveness of ceftazidime-avibactam as a treatment for multidrug resistant infections in a third level hospital.

Material and methods This was a before and after study in patients with multidrug resistant infections treated with ceftazidime-avibactam between April 2018 and April 2019. Those $<18$ years of age and patients who did not have the main study variables were excluded.

The main variable of our study was C reactive protein (CRP) before and after treatment. Secondary variables included age, sex, weight, dosage and isolated microorganism. An initial descriptive analysis was performed with mean (SD) or median (IQR, P25-P75) for numerical variables or absolute frequencies for nominal variables. For statistical analysis, the Wilcoxon test of paired measures was used to determine if there were differences in median CRP values before and after antibiotic treatment. The analyses were performed using the SPSS/PC statistical programme (V.24.0 for Windows, SPSS Inc, Chicago, Illinois, USA).

Results Thirty-six patients were treated with ceftazidime-avibactam from April 2018 to April 2019, of whom 32 were studied. Of these, 21 were men, average age was $63 \pm 11$ years and average weight was $71 \pm 20 \mathrm{~kg}$. The most common dosage was $2 \mathrm{~g}$ every 8 hours (25) and the most prevalent microorganism was Klebsiella pneumoniae (25).

The median initial CRP was $8.85 \mathrm{mg} / \mathrm{dL}(1.53-17.27)$ while the median final CRP was $3.29 \mathrm{mg} / \mathrm{dL}(0.59-6.78)$. Statistically significant differences were found in median CRP before and after antibiotic treatment $(\mathrm{z}=-3.35 ; \mathrm{p}=0.001)$.

Conclusion and relevance Ceftazidime-avibactam was found to be effective in patients presenting with multidrug resistant infections as it significantly reduced CRP, a marker used to monitor infections.

\section{REFERENCES AND/OR ACKNOWLEDGEMENTS}

No conflict of interest.

\section{PSQ-024 PHARMACOLOGIC INTERVENTION BY HOSPITAL PHARMACIST FOR LEUCOPENIA DUE TO TAZOBACTAM/PIPERACILLIN IN THE POSTPARTUM PERIOD: A CASE REPORT}

H Iwazawa*, T Tatsumichi, K Yamaguchi, H Ichinose, M Hariki, J Tomida, T Motoki, M Kaji, S Kosaka, H Houchi. Kagawa University Hospital, Department of Pharmacy, Kagawa, Japan

\subsection{6/ejhpharm-2020-eahpconf.341}

Background and importance Tazobactam/piperacillin (TAZ/ PIPC), indicated for pneumonia and intra-abdominal abscess in Japan, is recommended as a single drug therapy, together with carbapenems, in the guidelines for intra-abdominal infection published by the American College of Surgeons and Surgical Infection Society in 2010 in the USA. There are no reports of leucopenia after treatment with this drug in Japan.

Aim and objectives We observed the case of a postpartum woman who had leucopenia caused by TAZ/PIPC used for intra-abdominal infection. We have reported an improvement in symptoms owing to intervention by the hospital pharmacist.

Material and methods In our hospital, pharmacists are stationed in the maternity ward and share patient information at conferences held in other occupations once a week. A woman had continuous bleeding due to placental abruption after a normal delivery and underwent a total hysterectomy. On day 9, TAZ/PIPC was initiated as Bacteroides fragilis was found in a blood culture and was suspected to be causing intra-abdominal infection. A reduced white blood cell count persisted following the start of therapy, with leucopenia reported $\left(1.45 \times 10^{9} / \mu \mathrm{L}\right)$ on day 22 . As leucopenia was considered to be caused by TAZ/PIPC, we proposed discontinuation of the drug and the use of meropenem as an alternative. Leucopenia and intra-abdominal infection improved after switching to meropenem. On day 30, meropenem therapy was completed.

Results This patient had leucopenia on day 14 of treatment with TAZ/PIPC and her white blood cell count increased after drug discontinuation. We considered this event an adverse drug reaction caused by TAZ/PIPC, based on a previous report in which patients develop leucopenia, on average, on day 15 of TAZ/PIPC treatment. As the patient was in the postpartum period, we proposed meropenem as an alternative to allow the patient to continue to breast feed, because a lower proportion of this drug is transferred to breast milk. 